\title{
The microwave cavity perturbation technique for contact-free and in situ electrical conductivity measurements in catalysis and materials science $\dagger$
}

\author{
Maik Eichelbaum, ${ }^{* a}$ Reinhard Stößer, ${ }^{b}$ Andrey Karpov,${ }^{c}$ Cornelia-Katharina Dobner, ${ }^{c}$ \\ Frank Rosowski, ${ }^{c}$ Annette Trunschke ${ }^{a}$ and Robert Schlögl ${ }^{a}$
}

Received 12th August 2011, Accepted 16th November 2011

DOI: $10.1039 / \mathrm{clcp23462 \textrm {e }}$

We have developed a noncontact method to probe the electrical conductivity and complex permittivity of single and polycrystalline samples in a flow-through reactor in the temperature range of $20-500{ }^{\circ} \mathrm{C}$ and in various gas atmospheres. The method is based on the microwave cavity perturbation technique and allows the simultaneous measurement of microwave conductivity, permittivity and of the catalytic performance of heterogeneous catalysts without any need for contacting the sample with electrodes. The sensitivity of the method towards changes in bulk properties was proven by the investigation of characteristic first-order phase transitions of the ionic conductor rubidium nitrate in the temperature range between 20 and $320{ }^{\circ} \mathrm{C}$, and by studying the temperature dependence of the complex permittivity and conductivity of a niobium(v)-doped vanadium-phosphorous-oxide catalyst for the selective oxidation of $n$-butane to maleic anhydride. Simultaneously, the catalytic performance was probed by on line GC analysis of evolving product gases making the technique a real in situ method enabling the noninvasive investigation of electronic structure-function relationships.

\section{Introduction}

Electrical conductivity and complex permittivity measurements are important tools to reveal (electronic) structure-function relationships in materials science and heterogeneous catalysis. E.g., the reversible chemisorption of reactive gases on the surface of semiconductors such as metal oxides or chalcogenides can be accompanied by a changing conductance. This has been primarily ascribed to variations in the free charge carrier density in the conduction band (electrons) or in the valence band (electron holes) at the surface or within the bulk of the semiconducting sample. ${ }^{1}$ The dependence of the free charge carrier concentration, mobility, surface potential, or work function on the partial pressure of a certain gas has not only been used for the fabrication of selective gas sensors, but also to investigate and understand the electrical properties of semiconducting catalysts in operation. ${ }^{1,2}$ E.g., the rational design of heterogeneous bulk catalysts for the highly selective oxidation

${ }^{a}$ Fritz-Haber-Institut der Max-Planck-Gesellschaft, Abteilung für Anorganische Chemie, Faradayweg 4-6, D-14195 Berlin, Germany.E-mail: me@fhi-berlin.mpg.de;

Fax: + 49308413 4401; Tel: + 493084134566

${ }^{b}$ Humboldt-Universität zu Berlin, Institut für Chemie,

Brook-Taylor-Straße 2, D-12489 Berlin, Germany

${ }^{c} B A S F S E$, Chemicals Research and Engineering,

Carl-Bosch-Straße 38, D-67056 Ludwigshafen, Germany

$\dagger$ Electronic supplementary information (ESI) available: A powder

$\mathrm{X}$-ray diffractogram of the investigated Nb-VPO sample. See DOI:

$10.1039 / \mathrm{clcp} 23462 \mathrm{e}$ of alkanes to alkenes or oxygenates still suffers from a lack in understanding the nature, formation and working mode of active sites under industrially relevant conditions. The oxidation of one molecule $n$-butane to the important basic chemical maleic anhydride, e.g., demands in a single pass the abstraction of 8 hydrogen atoms, the insertion of 3 oxygen atoms, and the transfer of 14 electrons. It is still controversially debated, if such reactions can be explained on the basis of the single site concept with an isolated active catalytic center large enough to "store" reversibly the rather high amount of charge carriers transferred during a catalytic cycle, or if the bulk region underneath the active surface layer with appropriate charge transfer properties "buffering" the dynamic charge carrier concentrations on the surface has to be taken into account. ${ }^{3-10}$

To identify true correlations between conductivity, or generally any other property, of a sample and its catalytic activity and selectivity to a specific product or its functional capability as a selective gas sensor, the measurements have to be performed under in situ conditions. Usually, in-depth conductivity studies of heterogeneous catalysts under real working conditions are utilized by pressing the powder catalyst between two metal electrode discs or by the deposition of metal electrodes on catalyst pellets by means of physical vapor deposition. In such a way, e.g., the selective oxidation of $n$-butane on vanadium-phosphorous-oxide (VPO), ${ }^{4,5,11,12}$ of propane and propene on MoV mixed oxide catalysts, ${ }^{13,14}$ the oxidative coupling of methane ${ }^{15}$ or the oxidative dehydrogenation of butene on bismuth molybdates ${ }^{16}$ have been studied by measuring 
the $\mathrm{DC}$ or $\mathrm{AC}$ electrical resistance up to $10 \mathrm{MHz}$. However, the two-point contact method, which has been most often used in catalytic experiments, bears the severe disadvantage that it is highly dependent on the quality of the contacts between electrodes and catalyst. A bad contact and thus a high contact resistance (due to air gaps between electrode and material) would falsify the measured data significantly. In particular in the case of the typically used powder catalysts a good and reproducible contact is difficult, especially if the sample volume and/or geometry changes under reaction conditions due to sintering, water loss, etc. Four-point measurements could solve this problem, but are difficult to handle in a catalytic reactor. And even then electrode polarization and contact resistances at grain boundaries can complicate the data interpretation. Another typical concern in catalysis is the incorporation of catalytically active or inhibiting impurities from metal electrodes, especially since the most common electrode materials are platinum and iron.

In order to study charge transfer properties of materials and catalysts under working conditions we succeeded in developing a method based on the microwave cavity perturbation technique (MCPT) enabling the investigation of (di)electric properties of powders under operation in a contact-free and noninvasive manner, thus completely avoiding contact resistance and electrode-related problems. Our newly developed MCPT setup using the $\mathrm{TM}_{110}$ mode of a cylindrical silverplated brass cavity operating at $9.2 \mathrm{GHz}$ enables the measurement of the electrical conductivity and complex permittivity of single or polycrystalline samples at temperatures up to $500{ }^{\circ} \mathrm{C}$ in various gas atmospheres while on line probing gaseous reactants and products. MCPT was pioneered by Slater ${ }^{17}$ and relies on the adiabatic change of the characteristics (resonance frequency, quality factor) of a microwave cavity upon the introduction of the sample. MCPT has been successfully applied to conductivity and permittivity measurements of superconductors, superionic conductors, metals, semiconductors, and dielectric materials with high precision and in a broad range of frequencies. ${ }^{18,19}$ It was even shown that the state of automobile three-way-catalysts under lean and rich synthetic exhaust gas, respectively, can be monitored qualitatively by using the catalyst housing as microwave resonator and determining its resonance characteristics in situ. ${ }^{20,21}$ However, to the best of our knowledge MCPT has not been used to quantitatively determine the complex permittivity and conductivity of catalysts under working conditions, i.e. in a reactor at elevated temperatures in a reaction gas mixture while monitoring the catalytic performance.

The aim of this publication is to introduce the method of microwave cavity perturbation as a spectroscopic technique into the field of heterogeneous catalysis and to denote its applicability to quantitative and contact-free electrical conductivity measurements of powder samples in a flow-through reactor setup while simultaneously probing the catalytic performance under realistic working conditions. As a proof of principle the newly developed setup was calibrated with single crystals and powders of known complex permittivity, the firstorder phase transitions of the ionic conductor rubidium nitrate between 20 and $320^{\circ} \mathrm{C}$ were investigated, and the temperature dependence of both the conductivity and catalytic performance of a Nb-doped VPO catalyst (Nb-VPO) for the selective oxidation of $n$-butane to maleic anhydride was studied.

\section{Measuring electrical conductivity at microwave frequencies}

In polycrystalline, low-conductivity semiconductors consisting of conducting and insulating regions the transport of charge carriers is usually limited by the insulating barriers separating the conducting grains. ${ }^{22,23}$ Therefore, the DC conductivity $\sigma_{\mathrm{DC}}$ is determined mainly by the height and width of the intergrain barriers and not by the intrinsic electronic properties of the grains. In contrast, with AC (or microwave) measurements the grain boundaries become increasingly shunted by capacitive coupling between conducting grains with increasing frequency, and thus the contribution of the grain conductivity to the measured value increases. ${ }^{22,23}$ Alternatively, the conduction process can be described for some materials by a hopping mechanism, where electronic or ionic conduction is characterized on an atomistic scale by activated hopping of charge carriers between localized states. ${ }^{22-24}$ Both the barrier and the hopping model predict the following frequency dependence of the conductivity for a rather wide frequency range:

$$
\sigma(\omega)=\sigma_{\mathrm{DC}}+A_{\sigma} \omega^{s},
$$

with the material constant $s$ ranging from 0 to 2 (normally close to unity), the parameter $A_{\sigma}$, which is slightly dependent on temperature, and the angular frequency $\omega .^{22-24}$ Hence, while at lower frequencies the conductivity approaches the DC and thus intergrain conductivity, in the high frequency limit the bulk conductivity value (of the conducting grain in the barrier model) is approached. Measurements at microwave frequencies should therefore be well-suited for the investigation of polycrystalline materials such as typical heterogeneous catalysts excluding the disturbing influence of intergrain and electrode-grain contacts. Moreover, it is desirable to measure the conductivity over a broad frequency range to analyze systematically the electronic properties of grains, intergrain regions and the surface of the polycrystalline semiconductor and the influence of each on the catalytic performance.

A major advantage of the measurement at high frequencies is that the signals can be transmitted wireless between sender and receiver through air, and are consequently perfectly suited for noncontact methods. In principal, the interaction between radio- or microwaves and a material can be studied in a freefield antenna measurement setup or in a resonator. ${ }^{18}$ Since the formerly mentioned non-resonant method is only well-suited for planar sample geometries and the latter resonant technique is characterized by nearly no shape restrictions as well as a superior sensitivity, ${ }^{18,19,25,26}$ we have only considered the resonator technique for measuring electrical properties of catalysts.

As for measurements in a microwave resonator (cavity) one can distinguish between endplate perturbation, where the sample is equivalent to one resonator endplate, and enclosed perturbation, where the sample is placed in the center of the cavity (usually in the electric field maximum). ${ }^{17-19,25,26}$ Since in catalytic experiments it is undesirable to replace the resonator endplate by the catalyst, we have developed an in situ conductivity measurement technique for powder catalysts based 
on the microwave cavity enclosed perturbation technique, where the catalyst and the reactor tube are situated inside the resonator.

Usually, in a conventional MCPT setup a small sample situated in the center of the cavity induces only a small perturbation of the microwave resonance conditions. ${ }^{19,25,26}$ However, our approach demands the implementation of a complete quartz reactor including the catalytic sample into the cavity. Under such a strong perturbation of the electromagnetic fields inside the resonator the linear relationships between the (di-) electric material properties and the resonance frequency and quality factor of the filled resonator might break down. Another challenge is the high temperature needed for typical oxidation reactions, whereas the cavity has to be kept close to room temperature to avoid a degradation of the resonator wall surface and thus an irreproducible deterioration of the cavity's quality factor. ${ }^{18}$

We will show that by using a cylindrical X-band $\mathrm{TM}_{110}$ resonator originally designed for electron paramagnetic resonance experiments of particularly lossy samples, ${ }^{27}$ the changes in the resonance conditions, i.e. resonance frequency and quality factor, due to perturbation by the quartz reactor including the sample still depend linearly on the electronic properties, i.e. complex permittivity and conductivity, of the sample under investigation, allowing indeed quantitative measurements under reaction conditions.

\section{Experimental section}

\subsection{Materials}

The single crystal permittivity standards sapphire (0001), sapphire $(11 \overline{2} 0)$, rutile $(001)$, rutile (100) and lanthanum aluminate (100) were purchased from Crystal $\mathrm{GmbH}$ Berlin. Cylinders with a diameter of $3 \mathrm{~mm}(+0 /-0.1 \mathrm{~mm})$, a length of $5 \mathrm{~mm}$ and a lapped surface were prepared with an angular accuracy of $<0.5^{\circ}$. Selected samples were cut to obtain cylinders with a diameter of $3 \mathrm{~mm}$ and a length of about $1.2 \mathrm{~mm}$. Powder samples were prepared by milling selected single crystals with a planetary mill (Fritsch Pulverisette 7) and $\mathrm{Si}_{3} \mathrm{~N}_{4}$ milling bowls and balls.

For the in situ MCPT and differential scanning calorimetry thermoanalysis studies rubidium nitrate from Fluka with a purity grade of purum p.a., $>99 \%$ grade, was investigated.

As for the in situ catalysis and MCPT studies a $\mathrm{Nb}^{5+}$-doped vanadium-phosphorous-oxide catalyst with a molar $\mathrm{Nb} / \mathrm{V}$-ratio of 0.08 was prepared by the alcoholic route as described in the literature. ${ }^{28}$ Shortly, $\mathrm{V}_{2} \mathrm{O}_{5}$ was suspended into isobutanol containing $\mathrm{H}_{3} \mathrm{PO}_{4}$ obtaining a formal $\mathrm{P} / \mathrm{V}$ ratio of 1.15 . The dopant $\mathrm{Nb}^{5+}$ was included by solving niobium(v) ethoxide into the isobutanol $/ \mathrm{V}_{2} \mathrm{O}_{5}$ suspension with a $\mathrm{Nb} / \mathrm{V}$ ratio of 0.2 . The suspension was boiled under reflux for $16 \mathrm{~h}$. The solid was recovered by filtration and dried in vacuum at $150{ }^{\circ} \mathrm{C}$ for $16 \mathrm{~h}$ and calcined in air at $250{ }^{\circ} \mathrm{C}$ for $5 \mathrm{~h}$. According to element analysis (atomic emission spectrometry with inductively coupled plasma, Varian Vista Pro) the obtained catalyst precursor had a $\mathrm{Nb} / \mathrm{V}$ ratio of $0.08(\mathrm{~V}=27.2 \mathrm{wt} \%, \mathrm{Nb}=3.9 \mathrm{wt} \%)$. The catalyst precursor was pelletized employing $1 \mathrm{wt} \%$ graphite. The pellets were crushed and classified into a split fraction ranging from 0.5 to $1 \mathrm{~mm}$. The split was heated in air at $250{ }^{\circ} \mathrm{C}$ for
50 min, in $\mathrm{N}_{2} /$ air $/ \mathrm{H}_{2} \mathrm{O}=1 / 1 / 2$ at $370{ }^{\circ} \mathrm{C}$ for $5 \mathrm{~min}$ and finally in $\mathrm{N}_{2} / \mathrm{H}_{2} \mathrm{O}=1 / 1$ at $425^{\circ} \mathrm{C}$ for $195 \mathrm{~min}$. The thermally treated fresh catalyst had a bulk vanadium oxidation state of 4.26 as determined by potentiometric titration. The bulk catalyst was activated further for $60 \mathrm{~h}$ at reaction conditions $\left(400{ }^{\circ} \mathrm{C}\right.$, 2 vol\% $n$-butane, 3 vol\% $\mathrm{H}_{2} \mathrm{O}$ (1 ppm triethylphosphate), residual air, GHSV $=2000 \mathrm{~h}^{-1}$ ). As determined by X-ray diffractometry $\dagger$ (STOE Stadi-P transmission diffractometer using $\mathrm{CuK} \alpha$ radiation) the activated catalyst (referred to as $\mathrm{Nb}-\mathrm{VPO})$ contained as crystalline phases $52 \%(\mathrm{VO})_{2} \mathrm{P}_{2} \mathrm{O}_{7}$, $37 \% \alpha_{2}-\mathrm{VOPO}_{4}, 4 \% \quad \beta-\mathrm{VOPO}_{4}, 4 \% \mathrm{VOPO}_{4} \cdot 2 \mathrm{H}_{2} \mathrm{O}$, and $3 \%$ graphite. For the activated catalyst a BET surface area of $28.79 \mathrm{~m}^{2} \mathrm{~g}^{-1}$ was measured.

\subsection{Thermoanalysis}

Differential scanning calorimetry (DSC) thermoanalysis of rubidium nitrate powder was performed in $100 \mathrm{ml} \mathrm{min}^{-1} \mathrm{Ar}$ with a heating rate of $2 \mathrm{~K} \mathrm{~min}^{-1}$ in a Netzsch STA 449C Jupiter TG-DTA/DSC instrument.

\subsection{In situ MCPT setup}

As resonator a cylindrical $\mathrm{X}$-band $\mathrm{TM}_{110}$ silver-plated brass cavity (ZWG Berlin-Adlershof) with a height of $19.5 \mathrm{~mm}$ and a diameter of $38.5 \mathrm{~mm}$ was used. A description of a similar resonator type was given by Hyde. ${ }^{27}$ A quartz tube plug-flow reactor with $4 \mathrm{~mm}$ outer and $3 \mathrm{~mm}$ inner diameter containing the sample under investigation (powders were filled in with a bed height of $10 \mathrm{~mm}$ and embedded within quartz wool) and surrounded by a $10 \mathrm{~mm}$ outer diameter double-walled quartz dewar mantle was directly placed in the center of the cavity parallel to its endplates and perpendicular to the attached waveguide ( $c f$. Fig. 2). The quartz tube reactor was connected upstream to a gas delivery manifold equipped with mass flow controllers (Bronkhorst El-Flow) and downstream to an on line gas chromatograph (Agilent 7890A). For quantitative analysis the gas stream was split: in the first stream the gases $\mathrm{CO}_{2}, \mathrm{H}_{2} \mathrm{O}$, and $n$-butane were separated by a poraplot column, the gases $\mathrm{N}_{2}, \mathrm{O}_{2}$, and $\mathrm{CO}$ were separated by an additional molesieve column and detected by a thermoconductivity detector. In the second stream the product maleic anhydride was separated from the reaction mixture by a DB1 column and detected by a flame ionization detector. Transfer lines between reactor and $\mathrm{GC}$ were heated to $150{ }^{\circ} \mathrm{C}$ to avoid condensation of reaction products. Heating of the reactor was performed by preheating a stream of $81 \mathrm{~min}^{-1} \mathrm{~N}_{2}$ in a resistive furnace consisting of a Sylvania tungsten series I heater surrounded by a double-walled quartz dewar mantle. All dewar mantles were permanently evacuated by a turbomolecular pumping station (Pfeiffer HiCube 80 Eco) achieving a final pressure of about $10^{-7}$ mbar. The cavity was additionally cooled with two watercircuit-cooled copper plates attached to the resonator endplates.

The cavity was aperture coupled to a waveguide which was connected via coaxial cables to a vector network analyzer (Agilent PNA-L N5230C-225 operating between $10 \mathrm{MHz}$ and $20 \mathrm{GHz}$ and calibrated with the electronic calibration module Agilent N4691B ECal) in order to record resonance spectra of S11-parameters in reflection mode (reflected power versus frequency). All measurements were done with the cavity being 
critically coupled to the waveguide, accomplished by tuning the installed phase shifters and resonator aperture appropriately. The microwave power attenuation was set to $11 \mathrm{dBm}$. The resonance frequency was measured directly by determining the frequency at the minimum of the S11-parameter spectrum, the quality factor was determined by measuring the frequency difference of the resonance peak at a $3 \mathrm{~dB}$ power absorption level (full width at half maximum) and by multiplying the determined reciprocal bandwidth with the resonance frequency.

\section{Results and discussion}

\subsection{Development of the in situ MCPT setup}

The microwave cavity perturbation method is based on a small perturbation of the resonance conditions of a cavity upon introduction of a sample with given complex permittivity and/or electrical conductivity. Cavities can sustain specific standing wave modes, which are characterized by the resonance frequency $\nu_{0}$ (dependent on the cavity geometry) and the quality factor $Q_{0}$ (dependent on the geometry and the conductivity of the wall material) given by

$$
Q_{0}=\frac{\nu_{0}}{\Gamma_{0}},
$$

where $\Gamma_{0}$ denotes the full width at half maximum of the resonance absorption. After insertion of a small sample into the cavity the resonance frequency and the quality factor are changed to $\nu_{1}$ and $Q_{1}$, respectively:

$$
\begin{gathered}
\Delta \nu=\nu_{1}-\nu_{0}, \\
\frac{1}{Q_{1}}-\frac{1}{Q_{0}}=\frac{\Gamma_{1}}{\nu_{1}}-\frac{\Gamma_{0}}{\nu_{0}} .
\end{gathered}
$$

In the quasistatic and depolarization regime, where the penetration depth of the microwave field into a sample is much larger than the sample dimensions, the frequency and quality factor shifts can be directly related to the complex permittivity $\varepsilon=\varepsilon_{1}-i \varepsilon_{2}$ of the sample by the following approximations:

$$
\begin{aligned}
& \frac{\Delta \nu}{\nu_{0}}=A\left(\varepsilon_{1}-1\right) \frac{V_{\mathrm{s}}}{V_{\mathrm{c}}}, \\
& \frac{1}{Q_{1}}-\frac{1}{Q_{0}}=2 B \varepsilon_{2} \frac{V_{\mathrm{s}}}{V_{\mathrm{c}}},
\end{aligned}
$$

where $A$ and $B$ denote resonator constants depending on the cavity and sample geometry and resonance mode, and with $V_{\mathrm{s}}$ and $V_{\mathrm{c}}$ being the sample and cavity volume, respectively. ${ }^{18}$ In principle, $A$ and $B$ could be directly calculated, if the field distribution inside the cavity is known and does not change significantly upon the incorporation of the sample. However, for practical reasons the resonator constants are most often determined by measuring reference substances with known complex permittivities. For insulating and poorly conducting materials such as the catalysts of interest the quasistatic regime is a very good approximation, whereas for metal conductors the skin depth regime has to be accounted for. ${ }^{25}$

The relation between permittivity and electrical conductivity in the microwave range is not straightforward. For dielectric and insulating materials the imaginary part of the permittivity depends on the dielectric loss $\varepsilon_{2, \mathrm{dl}}$ (e.g. due to Debye relaxation) and ionic conductivity $\sigma_{\text {ion }}$ :

$$
\varepsilon_{2}=\varepsilon_{2, \mathrm{dl}}+\frac{\sigma_{\text {ion }}}{\omega \varepsilon_{0}},
$$

with $\varepsilon_{0}$ being the vacuum permittivity. ${ }^{18}$ As for dry semiconducting samples, the electrical conductivity $\sigma_{\text {AC }}$ (including electronic and ionic contributions) can be directly deduced from $\varepsilon_{2}$ measurements:

$$
\sigma_{\mathrm{AC}}=\omega \varepsilon_{0} \varepsilon_{2} .
$$

The precise assignment of the measured value for $\varepsilon_{2}$ to dielectric losses, ionic conductivity, and/or electronic conductivity is challenging. However, the different contributions differ in their dependencies on frequency, temperature, and chemical potentials of gases above the catalyst surface, and comprehensive studies of these influences should enable the discrimination between them.

As already mentioned in the introductory part, the design of the MCPT setup relies on the feasibility of the method to tolerate a heatable quartz flow reactor inside the resonator for catalytic studies. Comprehensive preinvestigations using a cylindrical $\mathrm{TE}_{011}$ cavity have shown that the conventional way of introducing the sample in the electric field antinode of an $\mathrm{X}$-band (i.e. $8-12 \mathrm{GHz}$ ) resonator is difficult, if the reactor setup has not to be extensively miniaturized (e.g. by using a Q-band dewar and quartz tube ${ }^{29}$ ) and a standard gas delivery and analysis system operating with $\mathrm{ml} \mathrm{min}{ }^{-1}$ rather than $\mu 1 \mathrm{~min}^{-1}$ gas flows has to be used. Thus, we investigated the $\mathrm{TM}_{110}$ mode of a cylindrical silver-plated brass resonator with a diameter of $38.5 \mathrm{~mm}$ and a height of $19.5 \mathrm{~mm}$ originally designed for the investigation of lossy samples in electron paramagnetic resonance spectroscopy. ${ }^{27}$ Generally, the electric fields inside a $\mathrm{TM}_{m n p}$ resonator $(m=0,1,2, \ldots ; n=1,2,3, \ldots$; $p=0,1,2, \ldots)$ for the radial $\left(E_{r}\right)$, angular $\left(E_{\phi}\right)$, and longitudinal $\left(E_{z}\right)$ components are given by the following equations:

$$
\begin{aligned}
& E_{r}=-\frac{a_{m n} p \pi}{R L} J_{m}^{\prime}\left[\frac{a_{m n}}{R} r\right] \cos [m \phi] \sin \left[\frac{p \pi}{L} z\right], \\
& E_{\phi}=\frac{1}{r} J_{m}\left[\frac{a_{m n}}{R} r\right] \frac{m p \pi}{L} \sin [m \phi] \sin \left[\frac{p \pi}{L} z\right], \\
& E_{z}=\left(\frac{a_{m n}}{R}\right)^{2} J_{m}\left[\frac{a_{m n}}{R} r\right] \cos [m \phi] \cos \left[\frac{p \pi}{L} z\right],
\end{aligned}
$$

with $R$ and $L$ being the radius and length of the cylindrical resonator, respectively, $J_{m}$ and $J_{m}^{\prime}$ the $m$ th order Bessel function and its first derivative, respectively, and $a_{m n}$ the zero of order $n$ of the $m$ th order Bessel function. ${ }^{18,30}$ The index $p$ is an integer giving the number of halfwaves along the cylinder axis. The resonance wavelength $\lambda_{0}$ for a $\mathrm{TM}_{m n p}$ mode is given by

$$
\lambda_{0}=\frac{2}{\sqrt{\left(\frac{a_{m n}}{R \pi}\right)^{2}+\left(\frac{p}{L}\right)^{2}}} .
$$

As for the $\mathrm{TM}_{110}$ mode only the longitudinal component $E_{z}$ is nonzero and the electric field becomes independent of the resonator length $L$ :

$$
E_{z}=\left(\frac{a_{11}}{R}\right)^{2} J_{1}\left[\frac{a_{11}}{R} r\right] \cos [\phi] .
$$




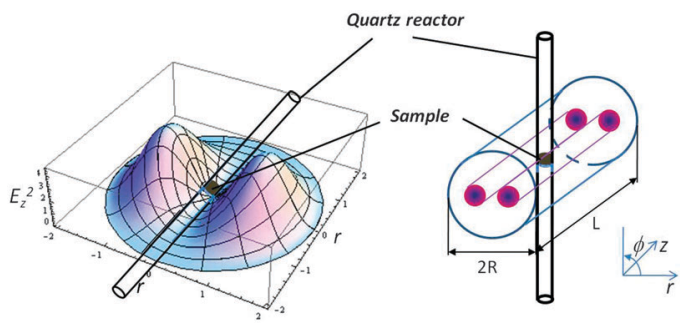

Fig. $1 \mathrm{TM}_{110}$ mode electric field distribution of a cylindrical $\mathrm{X}$ band cavity as calculated with eqn (13) and schematic representation of the implementation of a quartz tube reactor inside the resonator. The redviolet coils inside the drawn resonator indicate the electric field maxima.

The corresponding electric field distribution for a resonator with radius $R=2 \mathrm{~cm}$ is shown in Fig. 1. In our setup the quartz tube reactor with the sample is placed directly between the antinodes of the electric field as implied in the plot (Fig. 1). The thus decreased overall sensitivity can be accounted for by the usage of larger sample volumes that can be much easier to be handled and analyzed by conventional gas delivery and analysis equipment. As will be shown in the successive sections, a major advantage of the $\mathrm{TM}_{110}$ resonator is that it tolerates the implementation of a quartz reactor. For the measurement of catalysts under reaction conditions, i.e. at elevated temperatures, the quartz reactor tube with an external diameter of $4 \mathrm{~mm}$ and an inner diameter of $3 \mathrm{~mm}$ is sheathed by a permanently evacuated $\left(10^{-7} \mathrm{mbar}\right)$ double-walled quartz dewar vessel with an external diameter of $10 \mathrm{~mm}$ in order to maintain the temperature at the sample and to protect the resonator walls from heat radiation (Fig. 2). Additionally, the endplates of the cavity are cooled by a water-cooling-circuit. The heating of the sample is accomplished by preheating $\mathrm{N}_{2}$ gas in a resistance tungsten heater, which itself is sheathed by a permanently evacuated $\left(10^{-7} \mathrm{mbar}\right)$ double-walled quartz dewar vessel, connected upstream to the reactor tube. The preheated $\mathrm{N}_{2}$ gas is allowed to flow alongside the reactor tube, thus heating the sample inside. The tungsten heater is controlled by a PID circuit with a thermocouple situated inside the quartz reactor tube and directly upstream of the sample, but just outside of the resonator. The temperature differences between sample and thermocouple were determined in a control experiment and the measured temperatures in the

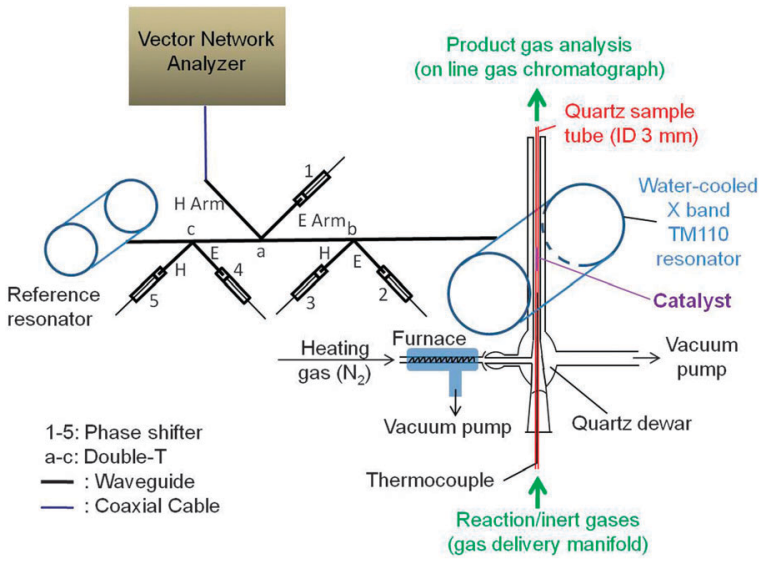

Fig. 2 Schematic of the in situ MCPT/on line GC setup. in situ experiments were corrected appropriately to the real values of the sample. The reactor tube itself is connected upstream to a gas delivery manifold and downstream to an on line gas chromatograph. A schematic of the reactor MCPT setup is depicted in Fig. 2. The calibrated reference resonator (resonance frequency can be tuned by changing the resonator length in micrometre steps) shown in the schematic can be used to measure independently the frequency of the microwave source.

\subsection{Calibration of the setup with single crystals and powders}

For the absolute determination of complex permittivities and electrical conductivities it is mandatory to calibrate the MCPT setup with single crystals of known permittivities. In order to cover a broad range of complex permittivities we investigated cylindrical single crystals of sapphire, rutile, and lanthanum aluminate. As for the anisotropic crystals of sapphire and rutile, we investigated in each case crystals with the cylindrical axes parallel and perpendicular to the optical axes ( $c$ axes), respectively, and thus the optical axes being perpendicular and parallel, respectively, to the electric field vector inside the resonator (single crystal cylinders were inserted perpendicular to the resonator axis and thus the electric field vector in our setup, $c f$. Fig. 1). All crystals had a diameter of $3 \mathrm{~mm}$ and a length of either 1.2 or $5 \mathrm{~mm}$ in order to fit perfectly into the quartz reactor tube and to be comparable with the shape of the later to be investigated catalytic samples.

In order to calibrate the setup the frequency and quality factor shifts due to the single crystals were measured and plotted versus the known real and imaginary part, respectively, of the permittivity according to eqn (5) and (6). By applying a least squares fitting (Levenberg-Marquardt algorithm; intercept was forced to go through zero) the slope and thus the calibration factors $A$ and $B$, respectively, were determined. The results for the $\varepsilon_{1}$ and $\varepsilon_{2}$ calibrations with single crystals are depicted in Fig. 3 and 4, respectively. It turned out that

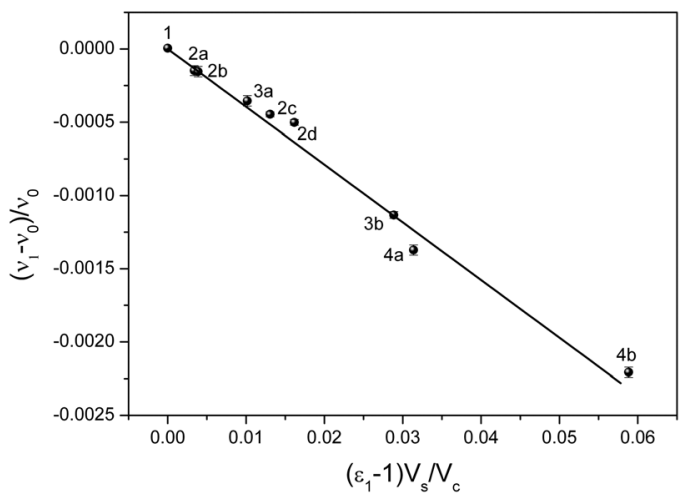

Fig. $3 \varepsilon_{1}$ calibration of the MCPT setup for different single crystals with a diameter of $3 \mathrm{~mm}$ and a length of either $5 \mathrm{~mm}$ (cylinders) or $1.2 \mathrm{~mm}$ (discs): 1 - empty quartz tube; $2 \mathrm{a}$ - sapphire disc, resonator axis perpendicular to optical axis $c ; 2 \mathrm{~b}$ - sapphire disc, axis parallel to $c$; 2c - sapphire cylinder, axis perpendicular to $c ; 2 \mathrm{~d}$ - sapphire cylinder, axis parallel to $c ; 3 \mathrm{a}$-lanthanum aluminate disc; $3 \mathrm{~b}$-lanthanum aluminate cylinder; $4 \mathrm{a}$ - rutile disc, axis perpendicular to $c ; 4 \mathrm{~b}$ - rutile disc, axis parallel to $c$. The straight line is the result of a least squares fitting (Levenberg-Marquardt algorithm) giving a slope $A$ of $-0.039 \pm 0.001$. 

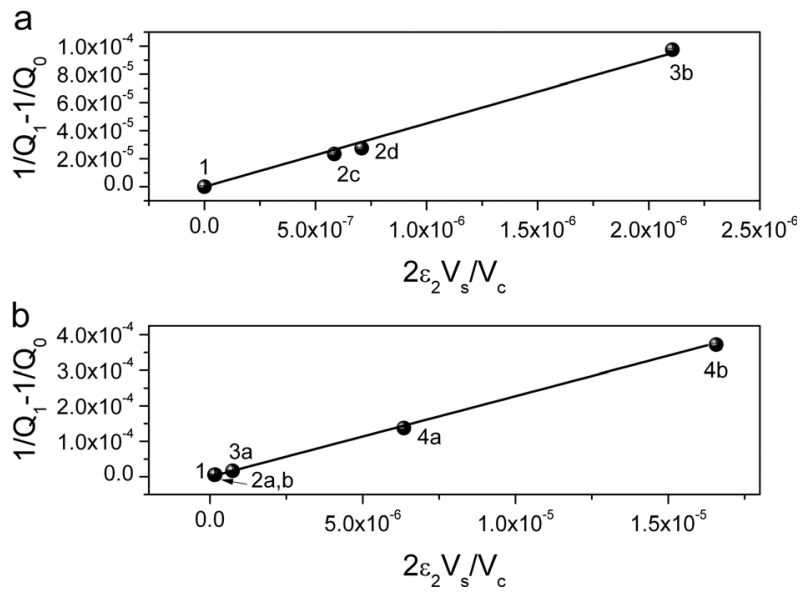

Fig. $4 \varepsilon_{2}$ calibration of the MCPT setup for different single crystals with a diameter of $3 \mathrm{~mm}$ in the form of cylinders with a length of $5 \mathrm{~mm}$ (a) and discs with a length of $1.2 \mathrm{~mm}$ (b): 1-empty quartz tube; 2a-sapphire disc, resonator axis perpendicular to optical axis $c$; $2 \mathrm{~b}$ - sapphire disc, axis parallel to $c ; 2 \mathrm{c}$ - sapphire cylinder, axis perpendicular to $c ; 2 \mathrm{~d}$-sapphire cylinder, axis parallel to $c$; 3a-lanthanum aluminate disc; 3b-lanthanum aluminate cylinder; $4 \mathrm{a}$ - rutile disc, axis perpendicular to $c ; 4 \mathrm{~b}$ — rutile disc, axis parallel to $c$. The straight lines are the results of least squares fittings (LevenbergMarquardt algorithm) giving slopes $B$ (cyl.) of $45 \pm 2$ (a) and $B$ (disc) of $22.7 \pm 0.2$ (b), respectively.

especially the quality factors depend significantly on the sample geometry, which is not unexpected due to non-homogeneous fields along the rather long cylinder axis. Consequently, two calibration constants $B$ have been determined for the cylinders with 1.2 and $5 \mathrm{~mm}$ length, respectively (Fig. 4).

With the determined calibration constants the complex permittivity of each single crystal sample was calculated again by using the actually obtained resonance frequency and quality factor shifts. The results are shown in Table 1 and are compared with reference values found in the literature and measured under similar conditions (room temperature, approx. 9-10 GHz) and given by the manufacturer of the single crystals.

After having proven that our MCPT reactor setup gives indeed a linear correlation between complex permittivities and both resonance frequency and quality factor shifts, a calibration curve for powder samples was recorded. For the correlation of the dielectric properties of bulk (single crystals) and polycrystalline samples (powders) the effective medium approximation as derived by Bruggeman ${ }^{31}$ for interspersions of spheres with the complex bulk permittivity $\varepsilon=\varepsilon_{1}-i \varepsilon_{2}$ and a volume fraction $\delta$ (packing fraction) into a medium with permittivity $\varepsilon_{m}$ (air with $\left.\varepsilon_{m}=1\right)$ was applied:

$$
\begin{gathered}
\frac{\varepsilon_{1}-\varepsilon_{1, \mathrm{p}}}{\varepsilon_{1, \mathrm{p}}^{1 / 3}}=(1-\delta)\left(\varepsilon_{1}-1\right), \\
\varepsilon_{2, \mathrm{p}}=\frac{3\left(\varepsilon_{1, \mathrm{p}}-1\right) \varepsilon_{1, \mathrm{p}}}{\left(\varepsilon_{1}-1\right)\left(\varepsilon_{1}+2 \varepsilon_{1, \mathrm{p}}\right)} \varepsilon_{2},
\end{gathered}
$$

where $\varepsilon_{1, \mathrm{p}}$ and $\varepsilon_{2, \mathrm{p}}$ represent the real and imaginary part, respectively, of the complex effective permittivity of the mixture (powder).

Single crystals of sapphire, rutile, and lanthanum aluminate were milled to fine powders and filled up to a filling height of $1 \mathrm{~cm}$ into the quartz tube reactor. As for the calibration for $\varepsilon_{1}$, the permittivity of the powder $\varepsilon_{1, \mathrm{p}}$ was calculated by eqn (14). In analogy to the calibration for single crystal samples, the real part of the calculated powder permittivities was plotted versus the frequency shift after

$$
\frac{\Delta \nu}{\nu_{0}}=A_{\mathrm{p}}\left(\varepsilon_{1, \mathrm{p}}-1\right) \frac{V_{\mathrm{s}}}{V_{\mathrm{c}}},
$$

to obtain the calibration constant $A_{\mathrm{p}}$ as the slope of the linear correlation. In order to proof the validity of this calibration procedure for a broader range of permittivities, powders with different packing fractions were prepared by pressing the powders with different pressures into the quartz tube. The results of the $\varepsilon_{1, \mathrm{p}}$ calibration are shown in Fig. 5.

Accordingly, $\varepsilon_{2, \mathrm{p}}$ was plotted versus the quality factor shift:

$$
\frac{1}{Q_{1}}-\frac{1}{Q_{0}}=2 B_{\mathrm{p}} \varepsilon_{2, \mathrm{p}} \frac{V_{\mathrm{s}}}{V_{\mathrm{c}}}
$$

The results of the calibration for $\varepsilon_{2, p}$ are depicted in Fig. 6 .

Both powder calibration constants are smaller, but are in the same order of magnitude as their single crystal counterparts. The deviations might come from the different volumes of the single crystals and the powders due to an inhomogeneous electric field along the sample tube as already observed for single crystal cylinders of different lengths. A summary of the complex permittivities of the different samples determined with the obtained calibration constants and their comparison with literature values is given in Table 1 .

As a result, it could be shown that with the newly developed MCPT reactor setup permittivities can be determined within a relative maximum error of approximately $10 \%$. Hence the

Table 1 Reference values for the complex permittivity of different single crystals (measured in the range of 9-10 GHz at room temperature), and of experimentally determined values for single crystal discs, cylinders (cyl.), and powder (p.) samples as measured by MCPT at 9.2 GHz and by

\begin{tabular}{|c|c|c|c|c|c|c|c|c|}
\hline Material & $\varepsilon_{1}^{32-35}$ & $\varepsilon_{1}($ disc $)$ & $\varepsilon_{1}$ (cyl.) & $\varepsilon_{1}($ p.) & $\varepsilon_{2}^{34-36}$ & $\varepsilon_{2}($ disc $)$ & $\varepsilon_{2}$ (cyl.) & $\varepsilon_{2}$ (p.) \\
\hline Sapphire $\| c$ & 11.6 & 11.5 & 9.2 & - & $2.3 \times 10^{-4}$ & $3.4 \times 10^{-4}$ & $2.0 \times 10^{-4}$ & - \\
\hline Sapphire $\perp \mathrm{c}$ & 9.4 & 10.2 & 8.2 & - & $1.9 \times 10^{-4}$ & $3.0 \times 10^{-4}$ & $1.6 \times 10^{-4}$ & - \\
\hline Sapphire (p.) & 10.5 & - & - & 10.5 & $2.1 \times 10^{-4}$ & - & - & $2.1 \times 10^{-4}$ \\
\hline $\mathrm{LaAlO}_{3}$ & 24.5 & 21.5 & 24.1 & 27.7 & $8.6 \times 10^{-4}$ & $8.4 \times 10^{-4}$ & $8.7 \times 10^{-4}$ & $9.8 \times 10^{-4}$ \\
\hline Rutile \|c & 173 & 163 & - & - & $2.4 \times 10^{-2}$ & $2.4 \times 10^{-2}$ & - & - \\
\hline Rutile $\perp \mathrm{c}$ & 89 & 97 & - & - & $8.9 \times 10^{-3}$ & $8.4 \times 10^{-3}$ & - & - \\
\hline Rutile (p.) & 130 & - & - & 118 & $1.6 \times 10^{-2}$ & - & - & $1.5 \times 10^{-2}$ \\
\hline
\end{tabular}
applying the calibration constants $A=-0.039, B$ (cyl.) $=45, B$ (disc) $=22.7, A_{\mathrm{p}}=-0.0266$, and $B_{\mathrm{p}}=36$ according to eqn (5), (6), (16) and (17), respectively. As for powder samples (samples with highest packing fraction) the bulk permittivities are given as recalculated by eqn (14) and (15). As for anisotropic samples, the mean value between $\varepsilon_{\|}$and $\varepsilon_{\perp}$ is given as reference value for powders 


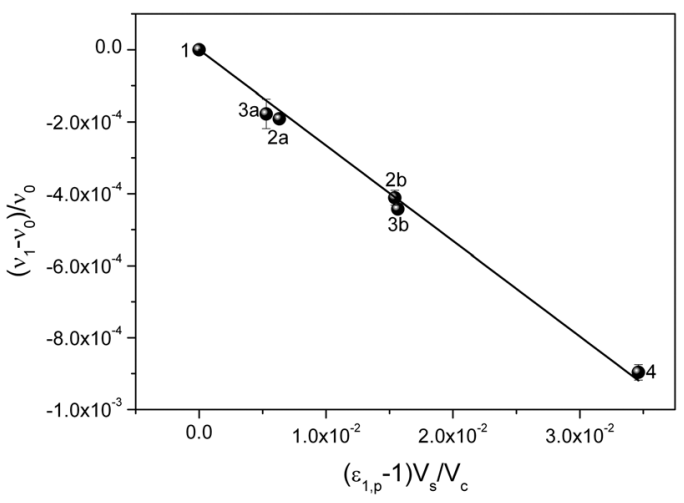

Fig. $5 \varepsilon_{1, \mathrm{p}}$ calibration of the MCPT setup for powders with different packing fractions: 1 - empty quartz tube; 2-sapphire, a: packing fraction 0.45 , b: 0.73; 3-lanthanum aluminate, a: 0.33, b: 0.57; 4-rutile, 0.60 . The straight line is the result of a least squares fitting (LevenbergMarquardt algorithm) giving a slope $A_{\mathrm{p}}$ of $-0.0266 \pm 0.0006$.

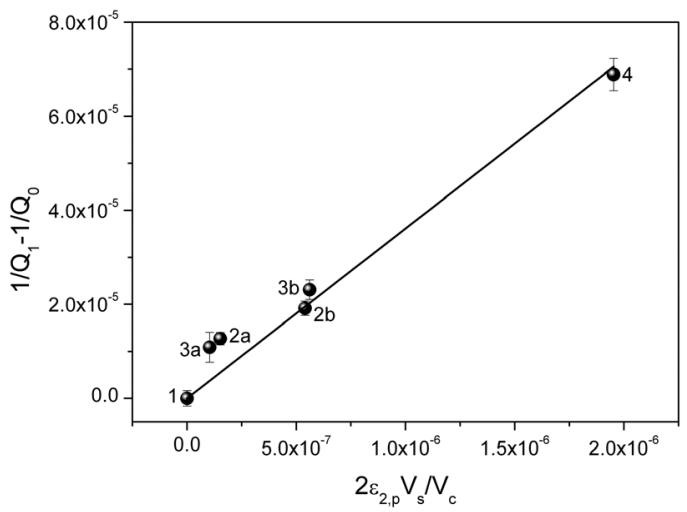

Fig. $6 \varepsilon_{2, \mathrm{p}}$ calibration of the MCPT setup for powders with different packing fractions: 1 -empty quartz tube; 2-sapphire, a: packing fraction 0.45 , b: $0.73 ; 3$-lanthanum aluminate, a: 0.33 , b: 0.57 ; 4 -rutile, 0.60 . The straight line is the result of a least squares fitting (Levenberg-Marquardt algorithm) giving a slope $B_{\mathrm{p}}$ of $36 \pm 2$.

MCPT reactor setup is indeed suitable for the quantitative determination of complex permittivities of both single crystals and powders despite the rather strong perturbation of the electric fields inside the cavity due to the implementation of a quartz tube reactor and a double-walled quartz dewar tube.

\subsection{In situ MCPT measurement of phase transformations (thermoanalysis) of $\mathrm{RbNO}_{3}$ between 20 and $320{ }^{\circ} \mathrm{C}$}

In a next step the method was tested for an in situ (thermoanalysis) experiment at elevated temperatures. For this purpose the ionic conductor rubidium nitrate was selected as interesting test material since it undergoes various phase transitions between room temperature and the melting point at $313{ }^{\circ} \mathrm{C}$. Starting at room temperature with a trigonal phase (IV) isomorphous with $\mathrm{CsNO}_{3}, \mathrm{RbNO}_{3}$ undergoes at $164^{\circ} \mathrm{C}$ a phase transformation to a cubic $\mathrm{CsCl}$ structure (III), at $219{ }^{\circ} \mathrm{C}$ to a rhomboedral (II) and at $285^{\circ} \mathrm{C}$ to a cubic $\mathrm{NaCl}$ phase (I). ${ }^{37-39}$ The appropriate phase transitions are related to orientational disorder of nitrate ions, and thus are accompanied by escalating changes in the complex permittivity. This was studied by MCPT at $9.2 \mathrm{GHz}$ with the calibrated reactor setup described before.
The rubidium nitrate powder was filled into the quartz reactor tube and melted to obtain a high packing density and a filling height of $1 \mathrm{~cm}$. After cooling, the sample was heated up again in static air to the appropriate temperatures with a ramp of $2 \mathrm{~K} \mathrm{~min}^{-1}$. Resonance frequencies and quality factors were measured after a holding time of 10 min under isothermal conditions after each temperature step. The real and imaginary part of the complex powder and bulk permittivity were determined in the investigated temperature range by calculating the frequency and quality factor shifts with respect to the values measured with an empty quartz tube under the same conditions and by applying eqn (14)-(17), and the resonator constants $A_{\mathrm{p}}$ and $B_{\mathrm{p}}$ obtained by the aforementioned calibration procedures. In addition, differential scanning calorimetry (DSC) was performed in the same temperature range to precisely determine the phase transformation temperatures. The data were compared with the obtained bulk permittivity values (Fig. 7). At room temperature a permittivity $\varepsilon_{1}$ of about 19 could be determined, which increases slightly up to 28 at $157^{\circ} \mathrm{C}$. The latter value is comparable to the tabulated $\varepsilon_{1}$ of 20 for single crystalline $\mathrm{RbNO}_{3}$ at $160{ }^{\circ} \mathrm{C}$ measured at $1 \mathrm{MHz} .{ }^{32}$ With increasing temperature three significant steps at 165, 220, and $290{ }^{\circ} \mathrm{C}$ in both $\varepsilon_{1}$ and $\varepsilon_{2}$ can be observed coinciding with the DSC minima at $166,223,284$, and $313{ }^{\circ} \mathrm{C}$ indicating endothermic phase transitions. According to the literature these steps and peaks, respectively, can be assigned to the IV-III, III-II, II-I phase transformations, and the melting point of $\mathrm{RbNO}_{3}$, respectively. ${ }^{37-39}$ The increase of $\varepsilon_{2}$ at the IV-III transition was assigned to a rise in the ionic conductivity

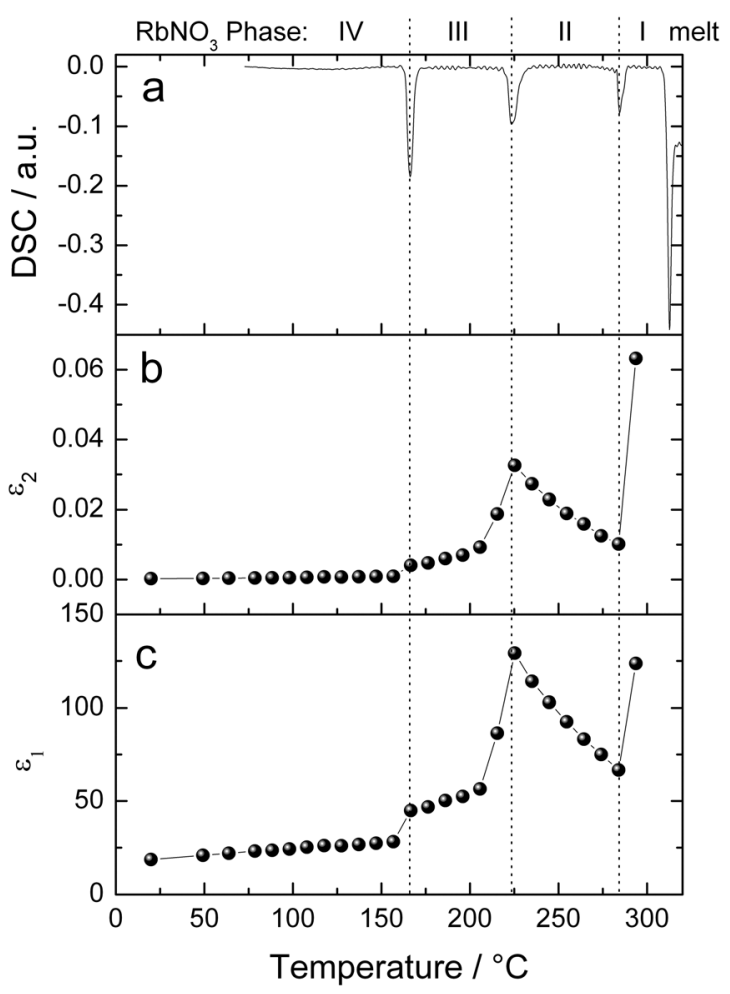

Fig. 7 (a) DSC graph of $\mathrm{RbNO}_{3}$ in $70 \mathrm{ml} \mathrm{min}-1$ argon measured at $2 \mathrm{~K} \mathrm{~min}^{-1}$. (b and c) Complex bulk permittivity of $\mathrm{RbNO}_{3}$ in static air measured at $9.2 \mathrm{GHz}$ by means of MCPT under isothermal conditions. The solid and broken lines are only a guide for the eye. 
(caused by cation Frenkel defects), which is after eqn (7) proportional to $\varepsilon_{2}$ if other dielectric losses can be neglected, caused by increased rubidium-nitrate ion distances in the crystal. Interestingly, just before the III-II transformation indicated by the appropriate endothermic DSC peak at $223{ }^{\circ} \mathrm{C}$ both $\varepsilon_{1}$ and $\varepsilon_{2}$ exhibit a sharp rise and a successive less steeper decline. The latter phenomenon was assigned to a decreased ionic conductivity due to smaller distances between rubidium and nitrate ions in the crystal. According to Kawashima and Uchiumi $^{38}$ the complex conductivity of a rubidium nitrate crystal measured by an AC impedance method at $1 \mathrm{kHz}$ along the $c$-axis undergoes a similar anomal trend, i.e. an initial increase followed by a decrease, though the decrease in both $\sigma_{1}$ and $\sigma_{2}$ (real and imaginary conductivity, respectively) was much faster than in our case. Unfortunately, the authors do not give an explanation for this behavior. The II-I phase transformation at $284{ }^{\circ} \mathrm{C}$ as indicated by the appropriate DSC peak overlaps with the beginning melting process of the sample. With melting the resonance absorption is getting very broad (due to an escalating ionic conductivity) with the consequence that the measurement of distinct permittivities becomes impossible. In summary, this experiment shows that the developed MCPT method is capable of monitoring bulk phase transformations in situ at elevated temperatures suggesting its potential as a thermoanalysis technique.

\subsection{In situ MCPT/on line GC measurement of a Nb-VPO catalyst for the selective oxidation of $n$-butane to maleic anhydride}

In the following section an in situ experiment on the permittivity and electrical conductivity of a niobium(v)-doped vanadiumphosphorous-oxide (Nb-VPO) catalyst with a molar $\mathrm{Nb} / \mathrm{V}$ ratio of 0.08 will be presented to show the capability of the developed MCPT setup to study the catalytic performance, microwave conductivity and their interrelation under realistic working conditions. As already mentioned in the Introduction VPO is well known to be a very good industrial catalyst for the selective oxidation of $n$-butane to maleic anhydride: ${ }^{3,4,7,9}$

$$
\mathrm{C}_{4} \mathrm{H}_{10}+\frac{7}{2} \mathrm{O}_{2} \rightarrow \mathrm{C}_{4} \mathrm{H}_{2} \mathrm{O}_{3}+4 \mathrm{H}_{2} \mathrm{O} .
$$

The only (and unwanted) by-products in significant amounts are $\mathrm{CO}$ and $\mathrm{CO}_{2}$. Furthermore, it was reported that $\mathrm{Nb}^{5+}$ can act as an $n$-type dopant upon increasing the activity of the catalyst. ${ }^{28}$ As already mentioned in the introductory remarks the selective oxidation of butane to maleic anhydride demands the transfer of 14 electrons and 3 oxygen atoms in a single pass. As a consequence it seems reasonable that a certain electrical conductivity is desirable for a high product yield. However, the total oxidation of one molecule $n$-butane to $\mathrm{CO}_{2}$ would require the transport of 26 electrons and 8 oxygen atoms, which would make an excessive conductivity unfavorable. Hence it is a plausible working hypothesis that the charge transfer properties (i.e. electrical conductivity in general, kind of charge carriers, charge carrier density, mobility, etc.) of a catalyst are important parameters that very probably influence both the overall activity and the selectivity to a desired product. The comprehensive investigation of the electrical conductivity of (redox) catalysts under working conditions is thus needed to understand and ideally improve (or rationally design) the electronic properties aiming at an optimum in the catalytic performance.

We studied a Nb-VPO catalyst which was activated for $60 \mathrm{~h}$ on stream under reaction conditions. The sample contained as major crystalline phase vanadyl pyrophosphate as proven by powder X-ray diffractometry. $\dagger$ For the MCPT measurements $75 \mathrm{mg}$ of a sieve fraction of 100-200 $\mu \mathrm{m}$ particles of Nb-VPO catalyst were filled into the quartz tube reactor obtaining a filling height of $1 \mathrm{~cm}$. Then, the temperature dependence of the complex permittivity was studied. First, the sample was heated up to $416{ }^{\circ} \mathrm{C}$ in $5 \mathrm{ml} \mathrm{min}^{-1}$ reaction mixture containing $2 \%$ $n$-butane, $20 \% \mathrm{O}_{2}$, and balance $\mathrm{N}_{2}$, corresponding to a weightbased hourly space velocity (WHSV) of $4000 \mathrm{ml} \mathrm{g}_{\mathrm{cat}}{ }^{-1} \mathrm{~h}^{-1}$, with a rate of $10 \mathrm{~K} \mathrm{~min}^{-1}$ to remove adsorbed water. After cooling down to room temperature, the catalyst was heated up again to the appropriate temperatures in the aforementioned reaction mixture with a ramp of $10 \mathrm{~K} \mathrm{~min}^{-1}$. The resonance frequencies and quality factors were measured after a holding time of 10 min under isothermal conditions after each temperature step. Simultaneously, the catalytic performance was monitored by probing the gas phase downstream of the catalyst by means of on line multidimensional gas chromatography (GC). To obtain the frequency and quality factor shifts and to calculate the temperature dependent complex bulk permittivity using eqn (14)-(17), respectively, the measurement was repeated with an empty quartz tube reactor to obtain the appropriate values for $\nu_{0}$ and $Q_{0}$ as reference. The results of this catalytic in situ $\mathrm{MCPT} /$ on line GC experiment are shown in Fig. 8. With increasing temperature an increase of both the real and imaginary part of the bulk permittivity was observed. $\varepsilon_{1}$ rises only slightly from 9 at $20^{\circ} \mathrm{C}$ to 12 at $416^{\circ} \mathrm{C}$. Hence no significant first-order phase changes can be observed in the regarded temperature range which could have been expected since the sample was activated and pretreated for $60 \mathrm{~h}$ under similar conditions. As for $\varepsilon_{2}$ a significant increase from 0.19 at room temperature to 0.77 at $416{ }^{\circ} \mathrm{C}$ can be recognized. As for the catalytic performance only above $300{ }^{\circ} \mathrm{C}$ the catalyst starts to convert $n$-butane to maleic anhydride as the main product with a selectivity of $70 \%$ at $400{ }^{\circ} \mathrm{C}$ (conversion of $26 \%$ ), and

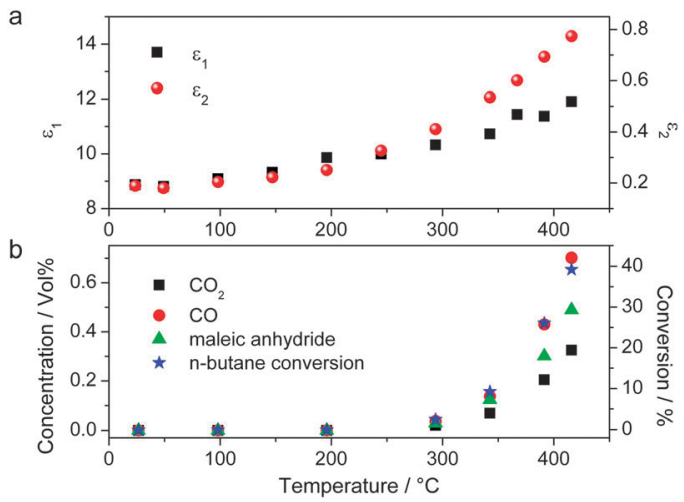

Fig. 8 (a) Temperature dependence of the complex bulk permittivity of a Nb-VPO catalyst as measured by in situ MCPT in a gas flow containing 2\% $n$-butane, $20 \% \mathrm{O}_{2}$ and balance $\mathrm{N}_{2}$ (WHSV: $4000 \mathrm{ml} \mathrm{g}_{\text {cat }}{ }^{-1} \mathrm{~h}$ ). (b) Simultaneously probed concentration of gases downstream of the reactor and relative $n$-butane conversion as measured by on line GC. 

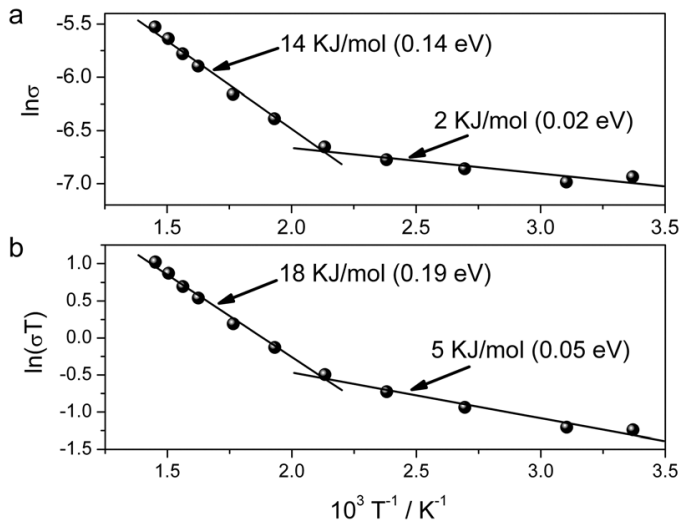

Fig. 9 Logarithmic Arrhenius-type plots of the microwave conductivity $\sigma$ of a Nb-VPO catalyst versus the reciprocal temperature. The lines are the results of linear least squares fittings (LevenbergMarquardt algorithm) and the shown values the thus obtained high and low temperature charge transport activation energies.

to $\mathrm{CO}$ and $\mathrm{CO}_{2}$ as minor products in agreement with the literature for a catalyst with moderate activity. ${ }^{3,4,7,9}$ Thus, we could indeed show that the newly developed in situ MCPT/on line GC setup permits the simultaneous contact-free measurement of complex permittivity (or conductivity) and catalytic performance in a flow-through reactor geometry.

To deduce a conductivity mechanism from the temperature dependence of the data the logarithm of the microwave conductivity $\sigma_{\mathrm{AC}}$ as calculated by eqn (8) was plotted versus the reciprocal temperature in an Arrhenius-type plot (Fig. 9a). As a result, two regimes with significantly different slopes can be identified. If the electrical conductivity $\sigma$ depends on temperature after

$$
\sigma=\sigma_{0} \exp \left(-\frac{E_{\mathrm{c}}}{k_{\mathrm{B}} T}\right)
$$

with the pre-exponential factor $\sigma_{0}, E_{\mathrm{c}}$ being the charge transport activation energy for electrical conduction (not to be confused with the Arrhenius activation energy of usual kinetic measurements in catalysis) and $k_{\mathrm{B}}$ the Boltzmann constant. The transport activation energy $E_{\mathrm{c}}$ can be calculated from the slope of the linear regression in the Arrhenius plot (Fig. 9a). ${ }^{9}$ A linear fit in the range between 200 to $416{ }^{\circ} \mathrm{C}$ gives a charge transport activation energy $E_{\mathrm{c}}$ of $140 \mathrm{meV}\left(14 \mathrm{~kJ} \mathrm{~mol}^{-1}\right)$. This value is below the DC activation energies found for vanadium(v) phosphates $\left(38.1-82.1 \mathrm{~kJ} \mathrm{~mol}^{-1}\right)^{12}$ and vanadyl pyrophosphate $\left(73.2-138.1 \mathrm{~kJ} \mathrm{~mol}^{-1}\right) .^{11}$ If the frequency dependence of $\sigma_{\mathrm{AC}}=A_{\sigma} \omega^{s}$ (cf. eqn (1)) and the Arrhenius ansatz (eqn (19)) are combined (while ignoring the slight temperature dependence of $A_{\sigma}$ ), the following frequency dependence of the charge transport activation energy would be obtained:

$$
E_{\mathrm{c}}=k_{\mathrm{B}} T\left(\ln \sigma_{0}-\ln \left(A_{\sigma} \omega^{s}\right)\right) .
$$

Hence a decreasing transport activation energy is expected with increasing frequency which could explain the rather low experimental values measured at $9.2 \mathrm{GHz}$ as compared to the DC measurements. ${ }^{2-24}$ This can be understood by the presumed sensitivity of DC measurements towards intergrain contacts with a rather high activation barrier, whereas high frequency measurements are supposed to probe more selectively the grain conductivity. Additionally, microwave losses due to dielectric relaxation from permanent molecular dipoles, ionic defects or relatively immobile electronic, polaronic or ionic charge carriers cannot be excluded, in particular at this rather high frequency measurement range. ${ }^{24}$ For the dipolar response of such rather immobile charges it is expected that the activation energy approaches zero. ${ }^{1}$ Thus the contribution of both dielectric relaxation and free charge carrier transport to the observed microwave conductivity (or $\varepsilon_{2}$ ) can explain the measured activation energy at 9.2 GHz.

In the region below $200{ }^{\circ} \mathrm{C}$ only a very small charge transport activation energy of $20 \mathrm{meV}\left(2 \mathrm{~kJ} \mathrm{~mol}^{-1}\right)$ is deduced from the Arrhenius plot (Fig. 9a). Herrmann et al. identified a similar trend while studying the DC conductivity of vanadyl pyrophosphate, i.e. a small temperature dependence at low temperatures. ${ }^{11}$ The determined "threshold" temperatures, i.e. the temperature separating the two regimes, ranged from 121 to $348{ }^{\circ} \mathrm{C}$ depending on the pretreatment. Furthermore, it was reported that vanadium phosphates such as $\mathrm{VOPO}_{4} \cdot 2 \mathrm{H}_{2} \mathrm{O}$, which was found as a minor phase in our Nb-VPO catalysts, $\dagger$ is a mixed proton-electron conductor, where protons are the dominating charge carriers at room temperature. ${ }^{40}$ In general, ionic conductivity can be described by the Nernst-Einstein relation:

$$
\sigma_{\text {ion }}=\frac{D(z e)^{2} c}{k_{\mathrm{B}} T},
$$

with $D$ being the diffusion constant, $c$ the concentration of ions with charge $z$, and $e$ the electronic charge. If both $D$ and $c$ are thermally activated, the temperature dependence of the conductivity can be written as:

$$
\sigma_{\text {ion }}(T)=\frac{\sigma_{0, \text { ion }}}{T} \exp \left(\frac{-E_{\mathrm{c}, \text { ion }}}{k_{\mathrm{B}} T}\right) .
$$

where in this case $E_{\mathrm{c} \text {,ion }}$ is the charge transport activation energy for ionic (e.g. proton) conduction. The graphical representation of $\ln (\sigma T)$ plotted versus $1 / T$ gives again two regimes (Fig. 9b). However, now in the low temperature region a charge transport activation energy of $50 \mathrm{meV}$ was found, which could be explained by surface proton conduction and/or by dielectric relaxation of adsorbed water or hydroxyl groups on the surface. The contribution of physisorbed water, hydroxyl groups and/or protons to the microwave absorption at lower temperatures is supported by thermogravimetric/mass spectroscopy (TG/MS) measurements. It could be shown that the original sample contains about $1 \mathrm{wt} \%$ water at room temperature, which is completely desorbed below $200{ }^{\circ} \mathrm{C}$ and contributes significantly to the measured conductivity (data not shown). As for the high temperature regime, a much higher transport activation energy of $190 \mathrm{meV}$ was found (Fig. 9b). In this regime electronic conduction might prevail. If the electronic conduction arises from the hopping of electrons or electron holes, respectively, among the mixed valence sites of the vanadium atoms (mainly $\mathrm{V}^{4+}$ and $\mathrm{V}^{5+}$ in VPO) as described by the small polaron theory, the same linear $\ln (\sigma T)$ dependence on $1 / T$ as given in eqn (22) would follow. ${ }^{22}$

However, the polaron theory was originally developed for amorphous or glassy materials or for impurity conduction in 
crystals only, ${ }^{22}$ and the exact determination of a conduction mechanism for crystalline phase pure VPO materials, in particular the contribution of the bulk, interfaces, and (sub)surface regions, needs much more research. Hence we will extend the MCPT measurements to lower frequencies in order to become even more sensitive to the charge transfer properties of the intergrain and surface regions of the studied materials. Furthermore, the determination of the exponent $s$ in eqn (1) for the dependence of the conductivity on frequency might pave a way to distinguish between mobile charge carrier polarisation and dipolar responses and to determine their relevance for catalysis. ${ }^{1,24}$ The advanced electronic characterization of the VPO catalyst, the comprehensive comparison of the microwave conductivity data with results from DC and $\mathrm{AC}$ impedance measurements as well as the interrelation of the conductivity results with the catalytic properties in order to describe the working mode of the catalyst would go beyond the scope of this manuscript, but will be published soon elsewhere. In particular the probing of the microwave conductivity in different gas atmospheres and the comparison with results from surface-sensitive techniques such as X-ray photoelectron and X-ray absorption spectroscopy (in dependence of the chemical potential of the gas phase) in order to determine the vanadium oxidation states on the surface and subsurface, a possibly formed space charge region or surface dipoles seems promising in providing deeper insights into the conductivity mechanisms of VPO catalysts. It was proposed that the small amount of $\mathrm{V}^{5+}$ usually found in $\left(\mathrm{V}^{4+} \mathrm{O}\right)_{2} \mathrm{P}_{2} \mathrm{O}_{7}$ acts as an acceptor dopant which can be formally described in the rigid band model in terms of an acceptor state situated right above the valence band. Upon thermal excitation of an electron from the valence band into the acceptor level electron holes are formed that can move freely in the valence band giving rise to the p-type conductivity found for VPO..$^{4,5,11,12}$ If all the aforementioned investigations are performed under operando conditions, which is work in progress, the (bulk and surface) electronic properties can be correlated indeed with the catalytic activity and selectivity.

\section{Conclusions}

Based on the microwave cavity perturbation technique (MCPT) we have developed a noncontact conductivity measurement method calibrated for the investigation of heterogeneous catalysts under absolutely realistic working conditions. MCPT relies on the adiabatic change of the characteristics (resonance frequency, quality factor) of a microwave resonator (cavity) upon the introduction of the sample under investigation, which enables the direct calculation of the complex permittivity and microwave conductivity of the sample. In contrast to conventional DC contact methods basically measuring intergrain contacts, noncontact MCPT probes grain (materials) properties. This represents a substantial advantage in view of the investigation of heterogeneously catalysed reactions at an atomistic or molecular level. The developed instrument consists of a quartz tube fixed-bed flow reactor inserted into an X-band $\mathrm{TM}_{110}$ microwave cavity operating at $9.2 \mathrm{GHz}$. The reactor tube is connected to an external furnace for preheating a gas stream to provide the desired temperatures at the sample, to a gas delivery manifold for inert and reaction gases, and to an on line GC gas analysis system allowing the simultaneous measurement of the catalytic performance. The apparatus was calibrated with various single crystals and powders with known complex permittivities. The method was successfully tested for studying first-order phase transformations of the ionic conductor rubidium nitrate. Additionally, the temperature dependence of the complex permittivity and electrical conductivity of a $\mathrm{Nb}^{5+}$-doped VPO catalyst for the selective oxidation of $n$-butane to maleic anhydride in a reaction gas mixture was investigated. As a result, two conductivity regimes, i.e. with a very small temperature dependence at lower and with an Arrhenius-type dependence at higher temperatures, were determined with the threshold temperature being $200{ }^{\circ} \mathrm{C}$. To our mind, this newly developed in situ MCPT technique bears the great potential to reveal interrelations between catalytic and electronic properties of materials under industrially relevant working conditions, thus offering the possibility to shed a new light on diverse still not fully understood heterogeneously catalyzed reactions.

\section{Acknowledgements}

The present work has been supported by the German Federal Ministry of Education and Research (BMBF) as part of the ReAlSelOx project (grant number: 033R028B).

\section{References}

1 A. Ovenston and J. R. Walls, J. Catal., 1993, 140, 464-480.

2 A. Gurlo and R. Riedel, Angew. Chem., Int. Ed., 2007, 46, 3826-3848.

3 G. Centi, Catal. Today, 1993, 16, 5-26.

4 J. M. M. Millet, Top. Catal., 2006, 38, 83-92.

5 P. J. Gellings and H. J. M. Bouwmeester, Catal. Today, 2000, 58, $1-53$.

6 R. K. Grasselli, Top. Catal., 2001, 15, 93-101.

7 G. J. Hutchings, J. Mater. Chem., 2004, 14, 3385-3395.

8 H. Bluhm, M. Hävecker, E. Kleimenov, A. Knop-Gericke, A. Liskowski, R. Schlögl and D. S. Su, Top. Catal., 2003, 23, 99-107.

9 J. M. Herrmann, Catal. Today, 2006, 112, 73-77.

10 J. C. Védrine, Top. Catal., 2002, 21, 97-106.

11 J. M. Herrmann, P. Vernoux, K. E. Béré and M. Abon, J. Catal., 1997, 167, 106-117.

12 F. Rouvet, J. M. Herrmann and J. C. Volta, J. Chem. Soc., Faraday Trans., 1994, 90, 1441-1448.

13 M. Caldararu, M. Scurtu, C. Hornoiu, C. Munteanu, T. Blasco and J. M. López Nieto, Catal. Today, 2010, 155, 311-318.

14 O. V. Safonova, B. Deniau and J. M. M. Millet, J. Phys. Chem. B, 2006, 110, 23962-23967.

15 Z. Zhang, X. E. Verykios and M. Baerns, Catal. Rev.:Sci. Eng., 1994, 36, 507-556.

16 D. A. G. van Oeffelen, J. H. C. van Hooff and G. C. A. Schuit, J. Catal., 1985, 95, 84-100.

17 J. C. Slater, Rev. Mod. Phys., 1946, 18, 441-512.

18 L.-F. Chen, C. K. Ong, C. P. Neo, V. V. Varadan and V. K. Varadan, Microwave electronics: Measurement and materials characterization, Wiley, 2004.

19 M. Dressel, O. Klein, S. Donovan and G. Grüner, Int. J. Infrared Millimeter Waves, 1993, 14, 2489-2517.

20 G. Fischerauer, M. Spörl, A. Gollwitzer, M. Wedemann and R. Moos, Frequenz, 2008, 62, 180-184.

21 R. Moos, M. Wedemann, M. Spörl, S. Reiss and G. Fischerauer, Top. Catal., 2009, 52, 2035-2040.

22 N. F. Mott and E. A. Davis, Electronic processes in non-crystalline materials, Clarendon Press, Oxford, 2nd edn, 1979. 
23 J. Sinkkonen, Phys. Status Solidi B, 1981, 103, 231-237.

24 A. K. Jonscher, Nature, 1977, 267, 673-679.

25 O. Klein, S. Donovan, M. Dressel and G. Grüner, Int. J. Infrared Millimeter Waves, 1993, 14, 2423-2457.

26 S. Donovan, O. Klein, M. Dressel, K. Holczer and G. Grüner, Int. J. Infrared Millimeter Waves, 1993, 14, 2459-2487.

27 J. S. Hyde, Rev. Sci. Instrum., 1972, 43, 629-631.

28 A. M. D. de Farias, W. de A. Gonzalez, P. G. P. de Oliveira, J. G. Eon, J. M. Herrmann, M. Aouine, S. Loridant and J. C. Volta, J. Catal., 2002, 208, 238-246.

29 R. Stösser, W. Herrmann, U. Marx and A. Brückner, J. Phys. Chem. A, 2011, 115, 2939-2952.

30 A. Frieser, Mikrowellenmesstechnik, Akademie-Verlag, Berlin, 1965.

31 D. A. G. Bruggeman, Ann. Phys., 1935, 24, 636-664.

32 CRC Handbook of Chemistry and Physics (Internet Edition), ed. W. M. Haynes and D. R. Lide, Taylor and Francis, 91st edn, 2011.
33 J. Krupka, K. Derzakowski, M. Tobar, J. Hartnett and R. G. Geyer, Meas. Sci. Technol., 1999, 10, 387-392.

34 N. M. Alford, J. Breeze, X. Wang, S. J. Penn, S. Dalla, S. J. Webb, N. Ljepojevic and X. Aupi, J. Eur. Ceram. Soc., 2001, 21, 2605-2611.

35 T. Konaka, M. Sato, H. Asano and S. Kubo, J. Supercond., 1991, 4, 283-288.

36 M. E. Tobar, J. Krupka, E. N. Ivanov and R. A. Woode, J. Appl. Phys., 1998, 83, 1604-1609.

37 R. Kawashima, K. Katsuki and K. Suzuki, J. Phys. Soc. Jpn., 1985, 54, 2057-2058.

38 R. Kawashima and T. Uchiumi, J. Phys. Soc. Jpn., 1986, 55, 3143-3150.

39 R. Kawashima and T. Uchiumi, Solid State Commun., 1986, 58, 625-627.

40 V. Zima, M. Vlček, L. Beneš, M. Casciola, L. Massinelli and R. Palombari, Chem. Mater., 1996, 8, 2505-2509. 